\title{
Exploring gaming as therapeutic care: A case study about the configuration of a game support for a pediatric hospital
}

\author{
Keywords \\ Children, Hospitalization, Innovation in Nursing, Nursing, Videogame.
}

Given the need for the hospitalisation of children and adolescents, bringing playfulness and playing can be strategies that help understand, promote, prevent, treat, and recover health. Playing is a childhood activity related to their growth and development, both motor, emotional, mental, and social. Play and games help in personal training and enhance skills. Thus, as each generation has its specificity, the current generation, more specifically that of children, has technology as a present and vital element in their daily lives. How children, the so-called digital natives, play nowadays is directly associated with technological means, which share space among traditional plays. In this sense, evidence suggests that interaction with electronic games stimulates brain activity and facilitates acceptance of the proposed treatment in the case of hospitalisation. It is undeniable that the illness and hospitalisation process brings emotional consequences to patients. Because of this, the nurse is an essential professional in implementing humanisation in the hospital context. However, promoting hospital humanisation also includes innovation in nursing. There are several types of inventions, including introducing new products or changes to some that are already on the market or even the approach of new methodological approaches to the search for design solutions, such as the use of Design Thinking. This approach aims to solve problems centred on the user, often through co-creation, in a collective and collaborative work of those involved with the problem to be solved.
Considering that hospital facilities often do not include the socialisation of all age groups, this thesis project aims to develop hospital support for video games, assessing the effectiveness of playful interventions, ergonomics, comfort, and patient accessibility. For the development of the product, one of the Design Thinking methodologies is being used: the Double Diamond, which is composed of the stages of discovery, definition, development, and implementation. In the discovery stage, the insight occurred through the follow-up of pediatric hospitalisation and was premised on speculations about "how to make the child's hospitalisation process smoother"? "Would it be possible to develop a hospital support for video games?". In the definition, a briefing was created to develop an efficient solution for the specific public, verifying the feasibility and available resources to carry out these actions. With the idea to be prototyped defined, a prototype of the support was designed based on the assumptions of feasibility, practicality, and functionality. The product under development was classified as an industrial design and deposited with the National Institute of Industrial Property for patent purposes. The developed support will be available for use by children in the hospital unit containing a monitor and video game to produce impacts as therapeutic care. The levels of entertainment and perceptions of health status will be evaluated. The Angels in Control project will generate a design outcome expected to provide more humanised and therapeutic care during pediatric hospitalisation. 